\title{
Classification of different degrees of adiposity in sedentary rats
}

\author{
A.S. Leopoldo ${ }^{1}$, A.P. Lima-Leopoldo ${ }^{1}$, A.F. Nascimento ${ }^{2}$, R.A.M. Luvizotto ${ }^{2}$, M.M. Sugizaki ${ }^{2}$, \\ D.H.S. Campos ${ }^{3}$, D.C.T. da Silva ${ }^{3}$, C.R. Padovani ${ }^{4}$ and A.C. Cicogna ${ }^{3}$ \\ ${ }^{1}$ Departamento de Desportos, Centro de Educação Física e Esportes, Universidade Federal do Espírito Santo, Vitória, ES, Brasil \\ ${ }^{2}$ Instituto de Ciências da Saúde, Universidade Federal do Mato Grosso, Sinop, MT, Brasil \\ ${ }^{3}$ Departamento de Clínica Médica, Faculdade de Medicina, Universidade Estadual Paulista, Botucatu, SP, Brasil \\ ${ }^{4}$ Departamento de Bioestatística, Instituto de Biociências, Universidade Estadual Paulista, Botucatu, SP, Brasil
}

\begin{abstract}
In experimental studies, several parameters, such as body weight, body mass index, adiposity index, and dual-energy X-ray absorptiometry, have commonly been used to demonstrate increased adiposity and investigate the mechanisms underlying obesity and sedentary lifestyles. However, these investigations have not classified the degree of adiposity nor defined adiposity categories for rats, such as normal, overweight, and obese. The aim of the study was to characterize the degree of adiposity in rats fed a high-fat diet using cluster analysis and to create adiposity intervals in an experimental model of obesity. Thirty-day-old male Wistar rats were fed a normal $(n=41)$ or a high-fat $(n=43)$ diet for 15 weeks. Obesity was defined based on the adiposity index; and the degree of adiposity was evaluated using cluster analysis. Cluster analysis allowed the rats to be classified into two groups (overweight and obese). The obese group displayed significantly higher total body fat and a higher adiposity index compared with those of the overweight group. No differences in systolic blood pressure or nonesterified fatty acid, glucose, total cholesterol, or triglyceride levels were observed between the obese and overweight groups. The adiposity index of the obese group was positively correlated with final body weight, total body fat, and leptin levels. Despite the classification of sedentary rats into overweight and obese groups, it was not possible to identify differences in the comorbidities between the two groups.
\end{abstract}

Key words: Obesity; Adiposity index; Rats; Cluster analysis

\section{Introduction}

Obesity is characterized by excessive body fat accumulation. It is considered a global epidemic and constitutes a major public health concern (1). Although the etiology of obesity is complex, certain risk factors have been implicated in its development, including increased caloric intake and physical inactivity (2). Recent investigations have demonstrated that obesity decreases life expectancy and is associated with numerous medical complications, such as type 2 diabetes mellitus, dyslipidemia, and cardiovascular disease (3). To better understand the physiopathology of abnormalities associated with obesity, various animal models using either genetic or dietetic approaches have been proposed $(4,5)$. Although genetic factors clearly contribute to the propensity of an individual to become obese, overconsumption of a highenergy diet may promote a positive energy balance and lead to overweight and obesity (6). Administering a high-fat diet to rodents reproduces many features of human obesity (7). Both humans and rodents tend to gain weight with high caloric intake (8). However, despite ad libitum feeding of a high-fat diet, obesity occurs in some but not all experiments $(9,10)$.

Fat storage in humans can be easily estimated using various indicators, such as waist circumference, the waist/hip ratio, skin-fold thickness, and bioimpedance (1). However, body mass index (the ratio of weight to height ${ }^{2}$ ) is most often used to quantify body fat in humans (11). In experimental studies in rats, several parameters, such as body weight (BW) $(12,13)$, body mass index (14), total body fat (15), fat pad mass $(15,16)$, adiposity index $(14,17)$, the Lee index $(18,19)$, magnetic resonance imaging (20), and dual-energy X-ray absorptiometry (21), have commonly been used to demonstrate increased adiposity and investigate the mechanisms underlying obesity. Studies have observed energy intake, nutrient partitioning, and weight gain to describe processes that differ between rats that are either prone or resistant to becoming obese $(22,23)$. However, these investigations have not experimentally classified the degree of adiposity because

Correspondence: A.S. Leopoldo: <andre.leopoldo@ufes.br> 
separating and identifying different adiposity intervals in animals is difficult, in contrast to classifying human obesity using the body mass index. Therefore, categories of adiposity in rats, such as normal, overweight, and obese, have not been defined in experimental studies.

Obtaining homogeneous groups of animals to measure their degree of adiposity is important, because it enables accurate diagnosis of experimental obesity and distinguishing between different parameters and comorbidities in these models. Because of the lack of studies that have classified the degrees of adiposity experimentally, the purpose of the present study was to characterize the level of adiposity in rats fed a high-fat diet (HFD) using the adiposity index. To achieve this objective, we used cluster analysis to identify the metabolic and nutritional profiles of the animals and create adiposity intervals in an experimental model of obesity. We hypothesized that a cluster analysis of data from rats fed a HFD for 15 weeks would allow us to distinguish various degrees of adiposity, similar to those defined in human obesity.

\section{Material and Methods}

\section{Animal care}

Thirty-day-old male Wistar rats were individually caged and subjected to different dietary regimens. All animals had free access to water and chow (50 g/day). After starting the experimental protocol, BW was recorded weekly. The environment was maintained under a 12-h light/dark cycle that started at 6:00 am, a clean-air room temperature of $23 \pm 3^{\circ} \mathrm{C}$, and $60 \pm 5 \%$ relative humidity. All experiments and procedures were performed in accordance with the Guide for the Care and Use of Laboratory Animals published by the U.S. National Institutes of Health and approved by the Ethics Committee of the Faculdade de Medicina, Universidade Estadual Paulista, Botucatu, SP, Brazil (\#565).

\section{Diet and experimental protocol}

The rats were distributed into two groups: normal diet (ND, $n=41)$ and HFD ( $n=43)$. The ND group was fed a standard diet containing $12.3 \% \mathrm{kcal}$ from fat, $57.9 \% \mathrm{kcal}$ from carbohydrates, and $29.8 \% \mathrm{kcal}$ from protein. The HFD group received a diet containing $49.2 \% \mathrm{kcal}$ from fat, $28.9 \% \mathrm{kcal}$ from carbohydrates, and $21.9 \% \mathrm{kcal}$ from protein. The HFD was calorically dense (HFD=3.65 kcal $/ \mathrm{g}$ vs $\mathrm{ND}=2.95 \mathrm{kcal} / \mathrm{g}$ ) because of the increased fat content. The rats were maintained on their respective diets for 15 consecutive weeks and then euthanized. After 15 weeks of the dietary protocol, the data underwent cluster analysis for adiposity classification.

\section{Experimental diet composition}

The experimental diets provided sufficient amounts of protein, vitamins, and minerals according to the Nutrient Requirements of Laboratory Animals (24). The ND and HFD used in this study were formulated by Agroceres (Brazil). The ingredients were first ground and then mixed with vitamins and minerals. The mixture was formed into pellets, dried in a ventilated drying oven at $55 \pm 5^{\circ} \mathrm{C}$, and stored at $-20^{\circ} \mathrm{C}$. The ND (RC Focus 1765) contained soybean oil, whole corn, wheat bran, soybean bran, dicalcium phosphate, sodium chloride, fish and meat flour, an antioxidant additive, and a vitamin and mineral mixture.

The HFD contained sodium chloride, casein, powdered milk, soybean protein concentrate, whole corn, cracker flour, dicalcium phosphate, calcium carbonate, emulsifier additives, antioxidants, flavoring, and a vitamin and mineral mixture. The HFD was formulated with a $0.9 \%$ concentration of sodium chloride and included saturated and unsaturated fatty acids that provided $20 \%$ and $80 \%$ of fat-derived calories, respectively. The high-unsaturated-fat diet consisted of $38 \%$ monounsaturated fatty acids and $42 \%$ polyunsaturated fatty acids, which were derived from the following fatty acids: $38 \%$ oleic acid, $41 \%$ linoleic acid $(n-6)$, and $1 \%$ linolenic acid (n-3).

\section{Adiposity index}

The animals were anesthetized with sodium pentobarbital (50 mg/kg BW, ip), decapitated, and thoracotomized. The adipose tissue fat pads were then dissected and weighed. Total body fat was measured as the sum of the following individual fat pad weights: epididymal fat + retroperitoneal fat + visceral fat. The adiposity index was calculated as (total body fat/final BW) $\times 100$ (25). The adiposity index was used as a measure of adiposity, because the degree of fat tends to increase gradually with obesity.

\section{Cluster analysis of the degree of adiposity}

Currently, there are no standard criteria for defining overweight or obesity in laboratory animals (e.g., rats and mice), companion animals, or other species. Using the adiposity index of animals fed a ND or HFD, we performed cluster analysis to establish similar groups with regard to adiposity level and to distinguish degrees of adiposity in these rats.

Cluster analysis is a statistical tool for classification and data reduction. This method allows large amounts of undivided data to be classified into subgroups based on similar characteristics (26). Methods such as k-nearest neighbor classification are useful exploratory tools for classifying relatively large amounts of data into subgroups when there is some prior knowledge of the potential number of subgroups within the given data set. For example, this method can be used to classify animals into either a normal-weight or obese group based on their phenotypic characteristics (e.g., BW) (27).

Using a set or unique parameter, the analysis generates a linkage tree that allows cases to be assigned to subsets, termed clusters. These clusters can then be grouped using the linkage method. A short linkage distance implies that the cases are similar. A long linkage distance implies that the cases are different. The method used in this study for data 
clustering was the closest neighbor technique (single linkage method), and the similarity coefficient used was the median Euclidean distance (26). The procedure used to analyze the conglomerates was the SAHN strategy for the formation of groups (SAHN involves "sequential, agglomerative, hierarchical, and non-overlapping" clusters). Finally, each cluster was described using dendrograms.

\section{Determination of groups following cluster analysis}

Following cluster analysis, the animals were allocated to groups according to their degree of adiposity. However, in biological experiments, particularly studies with animals, the results do not necessarily yield homogeneous groups (10). For this reason, the animals that received a HFD and exhibited changes in adiposity similar to the ND group were excluded from the analysis. The same procedure was performed with the rats that received a ND and exhibited changes in adiposity that were similar to the HFD group. These animals were rejected because of the necessity to characterize and distinguish adiposity from only obesity in rats that were fed a HFD and not in rats fed a balanced ND. After selecting the groups, the following characteristics were compared: nutritional state, systolic blood pressure (SBP), and metabolic and hormonal measures.

Nutritional analysis. Food consumption was measured daily, and BW was monitored once per week. Weekly caloric intake was calculated as the average weekly food consumption $\times$ the caloric value of each diet.

SBP. At the end of the experiment, SBP was assessed using the non-invasive tail-cuff method and a Narco BioSystems Electro-Sphygmomanometer (International Biomedical, USA). The average of two pressure readings was recorded for each animal.

Metabolic and hormonal measurements. At the end of the experimental period, the animals were subjected to $12-15 \mathrm{~h}$ of fasting, anesthetized using sodium pentobarbital (50 mg/kg BW, ip), and euthanized by decapitation. Blood samples were collected in dry tubes, and serum was separated by centrifugation at $3000 \mathrm{~g}$ for $15 \mathrm{~min}$ at $4^{\circ} \mathrm{C}$ and stored at $-80^{\circ} \mathrm{C}$ for subsequent analysis. The serum was analyzed for glucose (GL), triglycerides (TG), total cholesterol (T-Chol), non-esterified fatty acids (NEFAs), insulin, and leptin. Serum GL, TG, and T-Chol concentrations were measured using an enzymatic automatic analyzer system (Technicon, RA-XT System; Global Medical Instrumentation, USA). NEFA levels were determined according to the method of Johnson and Peters (28) using colorimetric kits (WAKO NEFA-C; Wako Pure Chemical Industries, Japan). Leptin and insulin levels were determined using an enzyme-linked immunosorbent assay with specific commercial kits according to the manufacturer's instructions (Linco Research, USA) (29).

\section{Statistical analysis}

Data from the cluster analysis, metabolic measurements, nutritional characteristics, and SBP values are reported as means $\pm S D$. These values are also reported as means and upper and lower limits of $95 \%$ confidence intervals for the mean. The results obtained from the cluster analysis were analyzed using one-way analysis of variance (ANOVA) followed by the Bonferroni post hoc test. Correlation analyses were conducted using the Pearson's linear correlation test. The level of significance was $5 \%$.

\section{Results}

Following cluster analysis, 12 animals were excluded from each group because these rats displayed adiposity indices that were similar to the opposing group (Figure 1). Thus, the present study comprised 29 control animals and 31 overweight or obese animals. Furthermore, the cluster analysis allowed us to divide the rats that were fed a HFD into overweight $(n=22)$ and obese $(n=9)$ groups according to their degree of adiposity (Figure 1 ).

Tables 1 and 2 demonstrate that the overweight and obese groups exhibited significant differences in food consumption, final BW, visceral, retroperitoneal and epididymal fat pad mass, total body fat, and the adiposity index compared with those of the ND group. Furthermore, serum GL, leptin, and insulin levels were higher in the overweight and obese groups compared with those of the ND group. However, no differences in SBP or NEFA levels were observed between groups (Table 2). Additionally, caloric intake and T-Chol and TG levels were lower in the ND group compared only with those of the obese group (Tables 1 and 2).

Figures 2 and 3 report the confidence intervals for each group. After comparing the overweight and obese groups with the ND group, we identified no overlapping confidence intervals for the adiposity index, total body fat, or final BW (Figure 2). The confidence intervals for SBP and TG levels overlapped between the ND, overweight, and obese groups. However, no overlap was observed for leptin, insulin, or GL between the overweight and ND groups (Figure 3). The leptin or T-Chol level confidence intervals in the obese group did not overlap with those of the ND group.

Obese animals displayed a significantly higher final BW $(P=0.03)$, total body fat $(P<0.001)$, and adiposity index $(P<0.001)$ than those of the overweight group (Tables 1 and 2). Additionally, fat pad weights were higher in the obese group than those in the overweight group $(P<0.001)$. Although food consumption did not differ between the overweight and obese groups $(P=0.076)$, caloric intake was greater in the obese group than that in the overweight group $(P=0.04)$. No differences were identified in SBP or NEFA, glucose, T-Chol, or TG levels between the overweight and obese groups.

The respective limits of the $95 \%$ confidence intervals are illustrated in Figures 2 and 3. No overlapping intervals for the adiposity index or total body fat were identified between the overweight and obese groups (Figure 2). However, overlapping confidence intervals for final BW, 


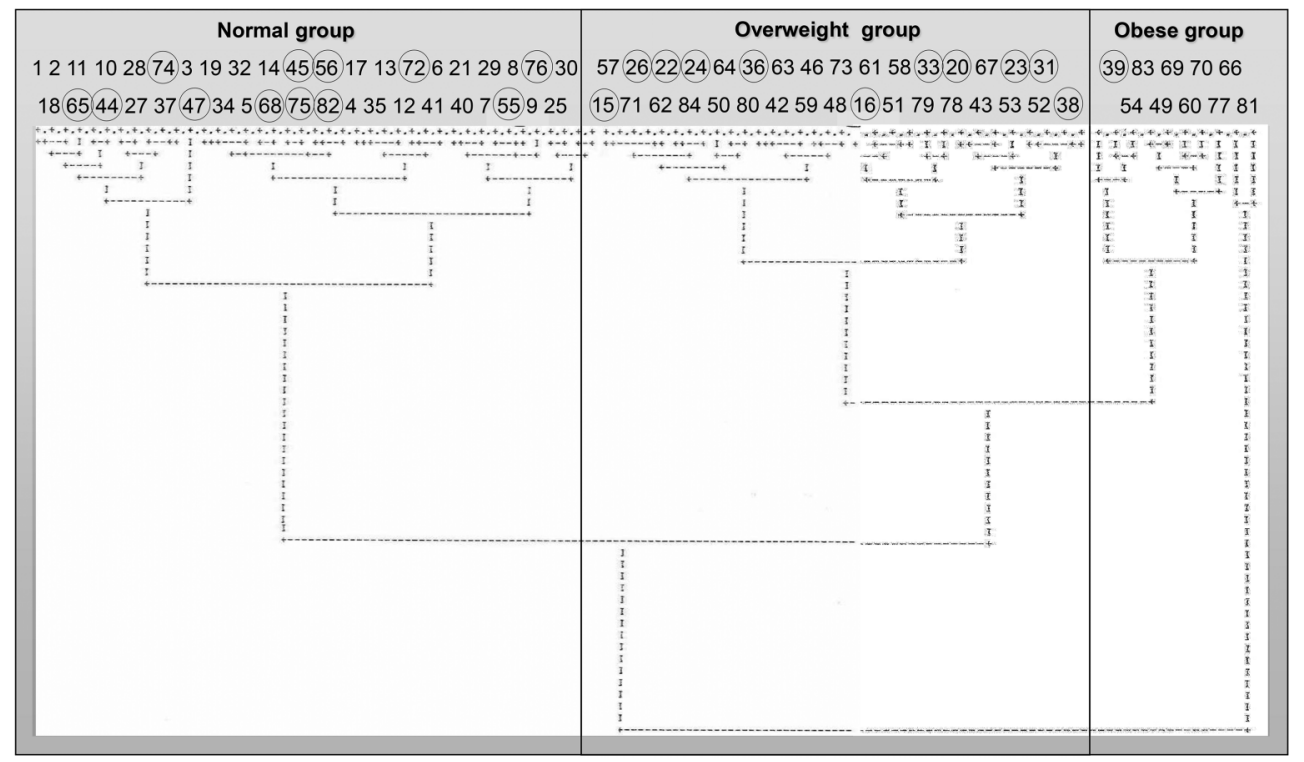

Figure 1. Dendrogram of cluster analysis according to the adiposity index of control, overweight, and obese rats. Normal: animals from 1 to 41 that were fed a standard diet; overweight and obese: animals from 42 to 84 that received a high-fat diet. Animals represented by a circle were excluded from the study.

SBP, and leptin, insulin, GL, T-Chol, and TG levels were observed between the two groups (Figures 2 and 3). The adiposity index for all rats was positively correlated with final BW $(r=0.67 ; P<0.05)$, body fat $(r=0.98 ; P<0.05)$, and leptin levels $(r=0.81 ; P<0.05)$ based on the Pearson's linear correlation test (Figure 4).

\section{Discussion}

The diet used in the present study was of sufficient nutritional density and duration to promote obesity in rats. The contribution of caloric intake to obesity development in this model elicited significant differences in BW, body fat, fat pad mass, and the adiposity index between the obese and ND groups. The HFD also promoted many indicators that resemble those of human comorbidities caused by obesity, such as hyperinsulinemia, hyperleptinemia, and elevated serum GL, T-Chol, and TG levels. These results are consistent with several studies that induced obesity in rats by HFD-feeding $(12,13,30)$.

The primary contribution of this study was the use of cluster analysis that allowed classification of degrees of adiposity in rats using the adiposity index, which is essential for studies that use experimental models to study obesity

Table 1. Nutritional parameters, fat pad mass and adiposity index.

\begin{tabular}{lccc}
\hline & \multicolumn{3}{c}{ Groups } \\
\cline { 2 - 4 } Variable & Normal $(\mathrm{n}=29)$ & Overweight $(\mathrm{n}=22)$ & Obese $(\mathrm{n}=9)$ \\
\hline $\mathrm{FC} \mathrm{(g/day)}$ & $27.6 \pm 1.8$ & $23.0 \pm 1.6^{*}$ & $24.7 \pm 2.8^{\#}$ \\
$\mathrm{Cl}($ Kcal/day) & $81.4 \pm 5.3$ & $83.8 \pm 5.8^{+}$ & $90.2 \pm 10.2^{\#}$ \\
$\mathrm{BW}(\mathrm{g})$ & $482 \pm 30$ & $512 \pm 25^{*+}$ & $547 \pm 56^{\#}$ \\
Visceral fat (g) & $5.74 \pm 1.24$ & $7.80 \pm 1.26^{*+}$ & $13.0 \pm 4.2^{\#}$ \\
Retroperitoneal fat $(\mathrm{g})$ & $7.78 \pm 1.28$ & $12.1 \pm 1.9^{*+}$ & $19.6 \pm 7.5^{\#}$ \\
Epididymal fat $(\mathrm{g})$ & $7.52 \pm 1.26$ & $10.6 \pm 2.0^{*+}$ & $16.0 \pm 4.3^{\#}$ \\
Body fat (g) & $21.0 \pm 2.9$ & $30.5 \pm 3.6^{*+}$ & $48.6 \pm 13.7^{\#}$ \\
Adiposity index (\%) & $4.36 \pm 0.49$ & $5.96 \pm 0.60^{*+}$ & $8.78 \pm 1.62^{\#}$ \\
\hline
\end{tabular}

Data are reported as means \pm SD. FC: food consumption; $\mathrm{Cl}$ : calorie intake; BW: body weight. Normal: group fed a standard diet; overweight and obese: groups fed a high-fat diet. ${ }^{*} \mathrm{P}<0.05$, overweight $v s$ normal; ${ }^{+} \mathrm{P}<0.05$, overweight vs obese; ${ }^{*} \mathrm{P}<0.05$, obese vs normal (one-way ANOVA; Bonferroni post hoc test). 
Table 2. Systolic blood pressure and metabolic and hormonal characteristics.

\begin{tabular}{lccc}
\hline & \multicolumn{3}{c}{ Groups } \\
\cline { 2 - 4 } Variable & Normal $(\mathrm{n}=29)$ & Overweight $(\mathrm{n}=22)$ & Obese $(\mathrm{n}=9)$ \\
\hline SBP $(\mathrm{mmHg})$ & $130 \pm 10$ & $130 \pm 11$ & $127 \pm 10$ \\
Leptin $(\mathrm{ng} / \mathrm{mL})$ & $3.17 \pm 0.92$ & $5.35 \pm 0.97^{*+}$ & $7.17 \pm 1.79^{\#}$ \\
Insulin $(\mathrm{ng} / \mathrm{mL})$ & $1.04 \pm 0.30$ & $1.41 \pm 0.33^{*}$ & $1.52 \pm 0.42^{\#}$ \\
NEFA $(\mathrm{mmol} / \mathrm{L})$ & $0.42 \pm 0.08$ & $0.47 \pm 0.11$ & $0.45 \pm 0.05$ \\
GL $(\mathrm{mg} / \mathrm{dL})$ & $156 \pm 22$ & $176 \pm 15^{*}$ & $178 \pm 13^{\#}$ \\
T-Chol $(\mathrm{mg} / \mathrm{dL})$ & $58.9 \pm 10.6$ & $65.7 \pm 11.6$ & $74.8 \pm 7.9^{\#}$ \\
TG $(\mathrm{mg} / \mathrm{dL})$ & $68.7 \pm 17.9$ & $76.1 \pm 17.4$ & $93.7 \pm 18.6^{\#}$ \\
\hline
\end{tabular}

Data are reported as means \pm SD. SBP: systolic blood pressure; NEFA: nonesterified fatty acid; GL: serum glucose; T-Chol: total cholesterol; TG: triacylglycerol. Normal: group fed a standard diet; overweight and obese: groups fed a high-fat diet. ${ }^{*} \mathrm{P}<0.05$, overweight vs normal; ${ }^{+} \mathrm{P}<0.05$ overweight vs obese; ${ }^{\#} \mathrm{P}<0.05$, obese vs normal (one-way ANOVA; Bonferroni post hoc test).

development. Cluster analysis is frequently used to stratify objects into groups according to similar characteristics $(26,27,31,32)$. To our knowledge, this is the first study that has used cluster analysis to experimentally classify adiposity in an animal model of obesity. Furthermore, this technique allowed the creation of classification groups in accordance with the level of adiposity, similar to those defined for human obesity (i.e., overweight and obese) using the body mass index. This method enabled us to investigate the effects of varying degrees of obesity on nutritional, metabolic and hormonal parameters.

In studies using obese rats, the classification of obesity severity is limited. Researchers usually separate animals into those that are prone or resistant to obesity development
(33-36), but they do not further sub-classify obesity-prone animals. In the present study, using the adiposity index, we demonstrated that obese rats exhibited different degrees of adiposity that could be classified into two groups. This heterogeneity is an important factor that may lead to discrepancies in experimental results because a relationship exists between the severity of obesity and its effects $(10,15)$. Seeking one criterion that can be used to classify animals according to obesity severity, similar to the body mass index in humans, is necessary.

Cluster analysis did not demonstrate overlapping adiposity indices or total body fat between the overweight and obese groups. Body weight, an index that is often used as an indicator of obesity, exhibited overlap between

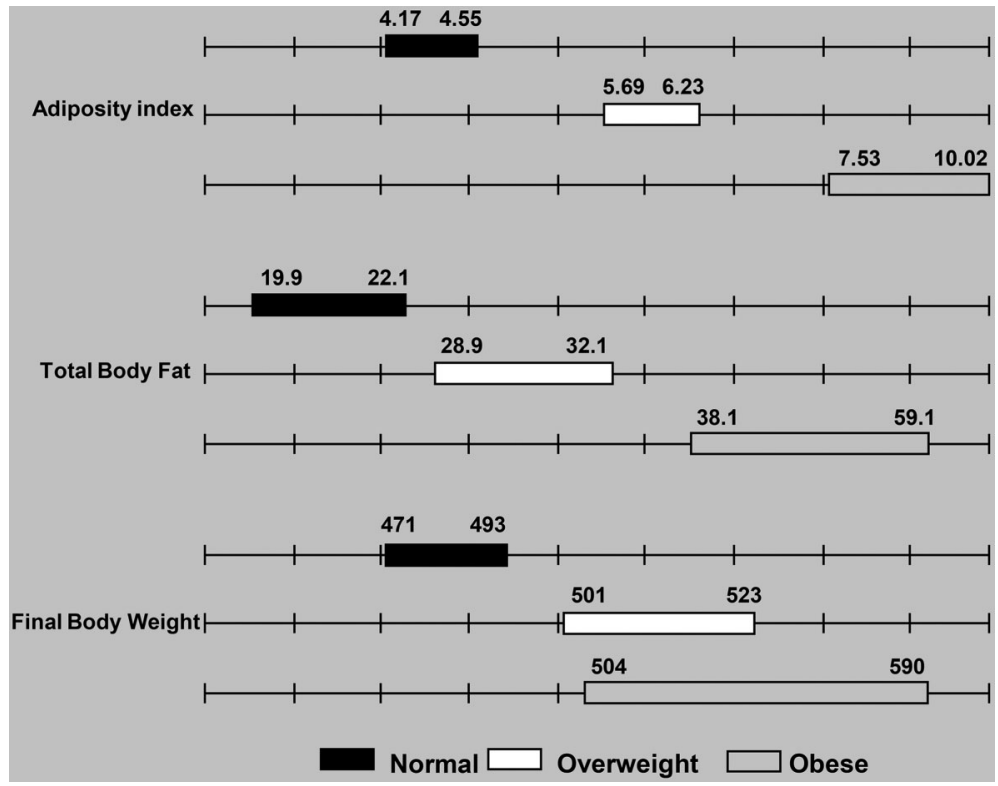

Figure 2. Confidence intervals of adiposity index $(\%)$, body fat $(\mathrm{g})$ and final body weight $(\mathrm{g})$ for normal, overweight, and obese rats, grouped according to the cluster analysis technique. Normal: group fed a standard diet; overweight and obese: groups fed a high-fat diet. 


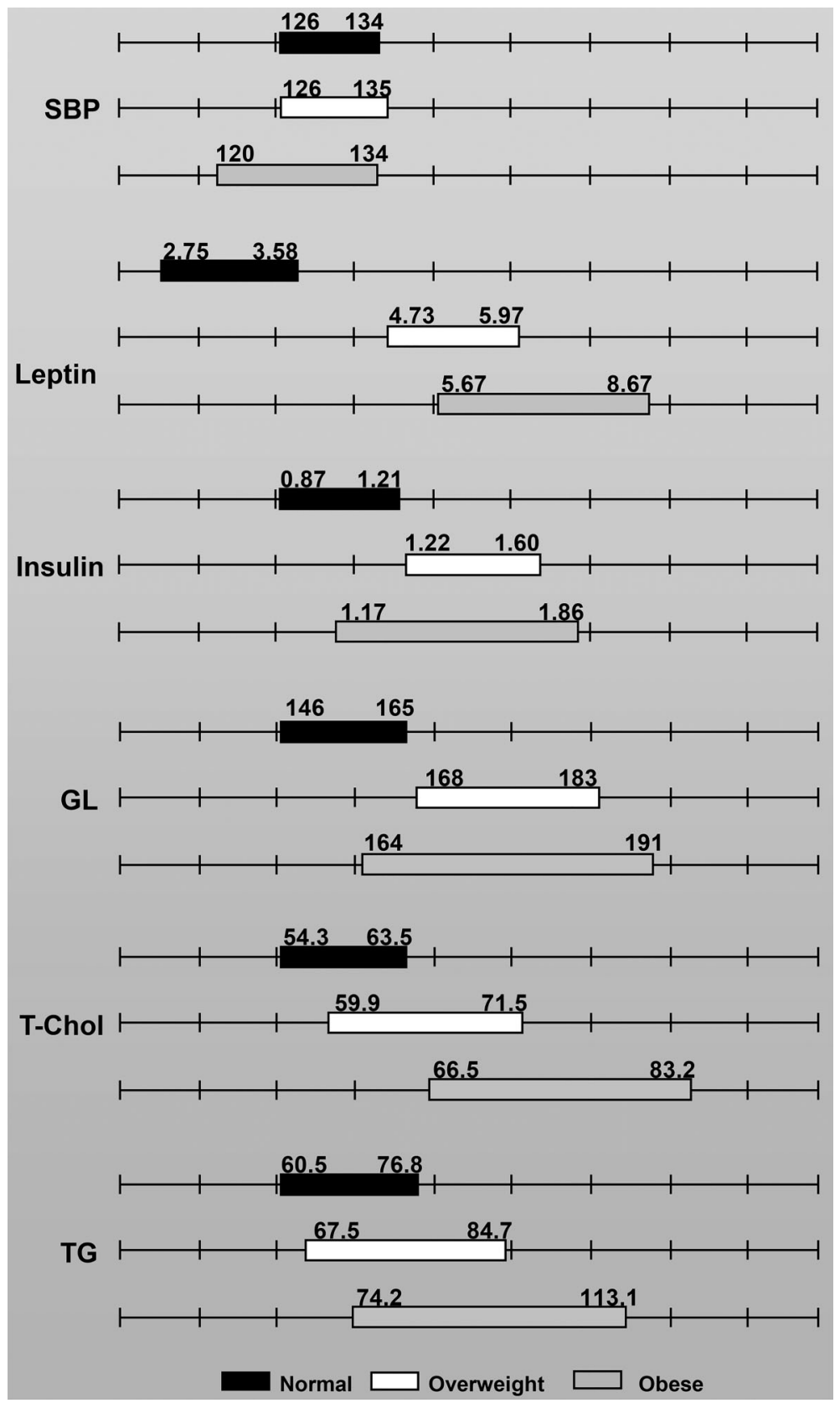

Figure 3. Confidence intervals of adiposity index and other variables for normal, overweight, and obese rats, grouped according to the cluster analysis technique. SBP: systolic blood pressure $(\mathrm{mmHg})$; Leptin ( $\mathrm{ng} / \mathrm{mL})$; Insulin $(\mathrm{ng} / \mathrm{mL}) ; \mathrm{GL}$ : glucose (mg/dL), T-Chol: total cholesterol (mg/dL); and TG: triacylglycerol (mg/dL). Normal: group fed a standard diet; overweight and obese: groups fed a high-fat diet.

the overweight and obese groups. However, the mean BW differed between the overweight and obese groups. Some previously published reports have demonstrated that BW can be a misleading indicator of obesity $(10,15)$. In this case, BW greatly underestimated the actual degree of obesity that developed in obese rats. Our overlapping BW results confirmed that this variable alone is not a good indicator of adiposity.

Obesity is accompanied by many metabolic and hormonal changes, including glucose, T-Chol, TG, leptin, and insulin levels $(12,13,30)$. However, in the present study the method used to classify overweight and obesity in rats was not effective at separating the metabolic and hormonal variables; insulin, leptin, GL, T-Chol, and TG confidence intervals overlapped between the two groups. Nevertheless, the mean leptin level was greater in the obese than in the overweight group. Previous studies have indicated that increased adiposity is directly associated with leptinemia $(37,38)$. Considering that leptin promotes lipolysis, reducing TG uptake by adipocytes (38), hyperleptinemia probably increased serum 

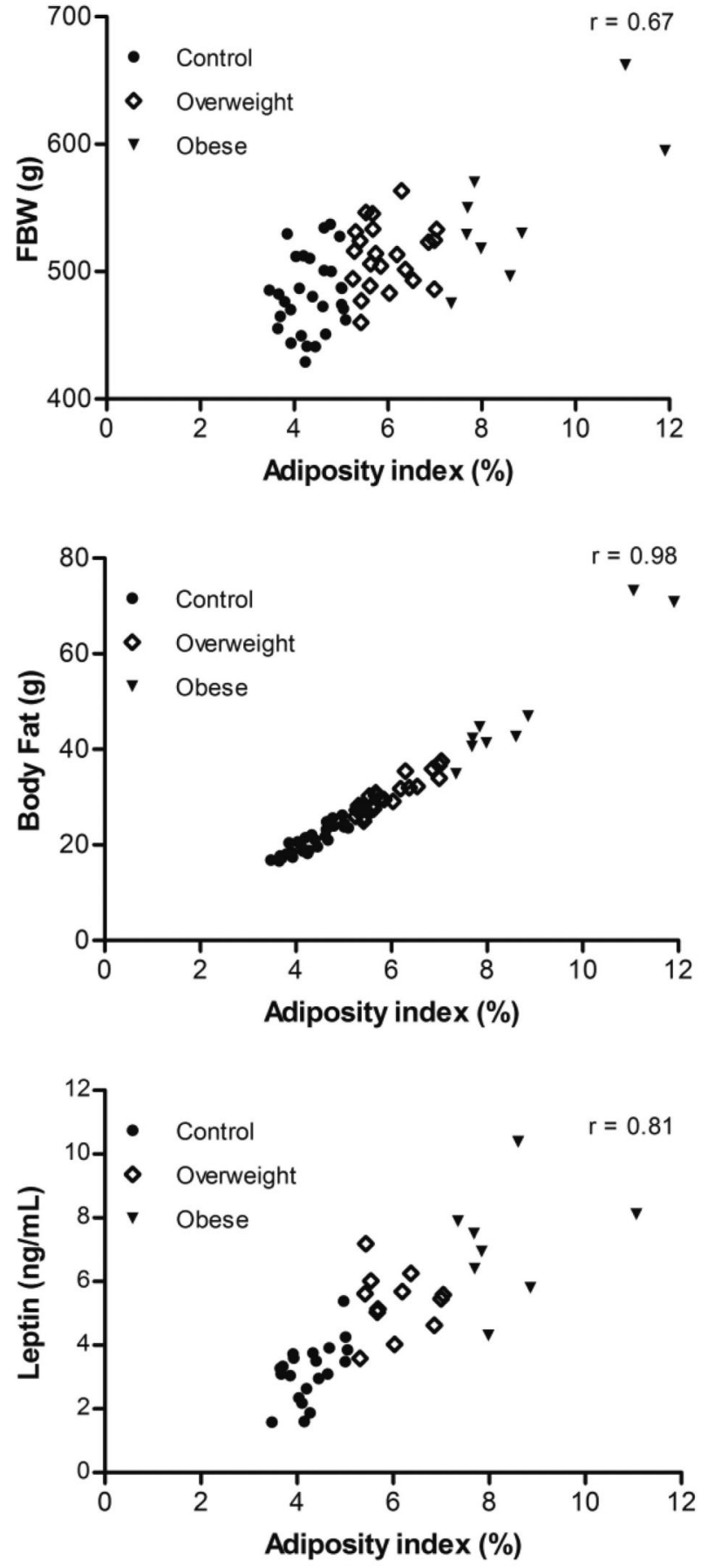

Figure 4. Correlations between adiposity index and final body weight (FBW), total body fat, and leptin levels after the 15-week experimental period.

TG levels in the obese rats. Importantly, hypertriacylglycerolemia is also associated with insulin resistance (38), which results in increased insulin levels to normalize blood glucose values, a condition called hyperinsulinemia.
Another parameter that is commonly impaired in obesity is arterial hypertension (19), although in this study alterations in SBP were not present. SBP confidence intervals also overlapped between the overweight and obese groups. Previous studies have reported an association between salt intake and increased blood pressure in experimental animals fed high levels of salt $(19,39,40)$. Dobrian et al. (19) demonstrated that both $2 \%$ and $4 \%$ $\mathrm{NaCl}$ in a HFD induced a rapid increase in SBP in obesityprone but not obesity-resistant or control rats. The absence of arterial hypertension in the overweight and obese groups in the current study may have been because of the low $\mathrm{NaCl}$ concentration $(0.9 \%)$ in the HFD. Because the confidence intervals of many variables (metabolic, hormonal, and cardiovascular) overlapped between the two groups, we can confirm that these parameters were not accurate as individual markers of the degree of obesity in rats. Therefore, the joint or isolated use of variables that do not overlap will allow the classification of obesity in rats into two different degrees of adiposity.

As described in the results section above, some variables overlapped between the normal, overweight, and obese groups, although the mean values were significantly different. Such overlap can occur because of the variability in animals, which results in non-homogenous groups. Thus, animals that were fed a ND exhibited variable responses, and some animals even displayed characteristics that resemble those of obese animals. Although overlapping confidence intervals occurred among the control, overweight, and obese groups, the results indicated that for the extreme points, the normal and obese group means differed for almost all variables, most likely because of the high degree of adiposity in the obese group. Only SBP and NEFA levels did not differ between the three groups. Our study also confirmed that the adiposity index and other markers of obesity were positively correlated (Figure 4). These results suggest that the adiposity index is a good indicator of obesity, similar to several other studies that have used the adiposity index as an indicator of fat accumulation $(14,19,36)$.

In conclusion, cluster analysis of the data obtained from sedentary rats subjected to 15 weeks of a HFD allowed the rats to be divided into two different categories of adiposity (overweight and obese). However, identifying differences in the cardiac and metabolic parameters between these two groups was not possible. Further studies with more prolonged periods of obesity are necessary to evaluate the different degrees of obesity and their potential association with disorders that resemble human comorbidities.

\section{Acknowledgments}

This manuscript has been proofread and edited by native English speakers with related biomedical backgrounds from BioMed Proofreading LLC. This research was supported by FAPESP (\#2007/53267-3 and \#2006/ 59485-0). 


\section{References}

1. O'Brien PE, Dixon JB. The extent of the problem of obesity. Am J Surg 2002; 184: 4S-8S.

2. Stein CJ, Colditz GA. The epidemic of obesity. J Clin Endocrinol Metab 2004; 89: 2522-2525.

3. López-Jiménez F, Cortés-Bergoderi M. Obesity and the heart. Rev Esp Cardiol 2011; 64: 140-149, doi: 10.1016/j.recesp. 2010.10.010.

4. Carroll JF, Dwyer TM, Grady AW, Reinhart GA, Montani JP, Cockrell K, et al. Hypertension, cardiac hypertrophy, and neurohumoral activity in a new animal model of obesity. Am J Physiol 1996; 271: H373-H378.

5. Kanasaki K, Koya D. Biology of obesity: lessons from animal models of obesity. J Biomed Biotechnol 2011; 2011: 197636, doi: 10.1155/2011/197636.

6. Swinburn B, Egger G. Preventive strategies against weight gain and obesity. Obes Rev 2002; 3: 289-301, doi: 10.1046/ j.1467-789X.2002.00082.x.

7. Lauterio TJ, Bond JP, Ulman EA. Development and characterization of a purified diet to identify obesity-susceptible and resistant rat populations. J Nutr 1994; 124: 2172-2178.

8. Chicco A, Bernal C, Soria A, Giangrossi G, Lombardo Y. Dietary effects of partial or total substitution of sucrose for starch on glucose and lipid metabolism in dyslipidemic rats. Nutr Res 1999; 19: 281-293, doi: 10.1016/S0271-5317(98)00191-2.

9. Commerford SR, Pagliassotti MJ, Melby CL, Wei Y, Gayles EC, Hill JO. Fat oxidation, lipolysis, and free fatty acid cycling in obesity-prone and obesity-resistant rats. Am J Physiol Endocrinol Metab 2000; 279: E875-E885.

10. Nascimento AF, Sugizaki MM, Leopoldo AS, Lima-Leopoldo AP, Nogueira CR, Novelli EL, et al. Misclassification probability as obese or lean in hypercaloric and normocaloric diet. Biol Res 2008; 41: 253-259, doi: 10.4067/S071697602008000300002.

11. WHO. Obesity: preventing and managing the global epidemic. Report of a WHO consultation. World Health Organ Tech Rep Ser 2000; 894: 1-253.

12. Akiyama T, Tachibana I, Shirohara $\mathrm{H}$, Watanabe $\mathrm{N}$, Otsuki M. High-fat hypercaloric diet induces obesity, glucose intolerance and hyperlipidemia in normal adult male Wistar rat. Diabetes Res Clin Pract 1996; 31: 27-35, doi: 10.1016/ 0168-8227(96)01205-3.

13. Wilson CR, Tran MK, Salazar KL, Young ME, Taegtmeyer $H$. Western diet, but not high fat diet, causes derangements of fatty acid metabolism and contractile dysfunction in the heart of Wistar rats. Biochem J 2007; 406: 457-467.

14. Sinitskaya N, Gourmelen S, Schuster-Klein C, GuardiolaLemaitre $B$, Pevet $P$, Challet $E$. Increasing the fat-tocarbohydrate ratio in a high-fat diet prevents the development of obesity but not a prediabetic state in rats. Clin Sci 2007; 113: 417-425, doi: 10.1042/CS20070182.

15. Woods SC, Seeley RJ, Rushing PA, D’Alessio D, Tso P. A controlled high-fat diet induces an obese syndrome in rats. J Nutr 2003; 133: 1081-1087.

16. Song Y, Park HJ, Kang SN, Jang SH, Lee SJ, Ko YG, et al. Blueberry peel extracts inhibit adipogenesis in 3T3-L1 cells and reduce high-fat diet-induced obesity. PLoS One 2013; 8: e69925, doi: 10.1371/journal.pone.0069925.
17. Levin BE, Dunn-Meynell AA, McMinn JE, Alperovich M, Cunningham-Bussel A, Chua SC Jr. A new obesity-prone, glucose-intolerant rat strain (F.DIO). Am J Physiol Regul Integr Comp Physiol 2003; 285: R1184-R1191, doi: 10.1152/ ajpregu.00267.2003.

18. Diniz YS, Burneiko RM, Seiva FR, Almeida FQ, Galhardi $\mathrm{CM}$, Filho JL, et al. Diet compounds, glycemic index and obesity-related cardiac effects. Int J Cardiol 2008; 124: 92-99, doi: 10.1016/j.jijcard.2006.12.025.

19. Dobrian AD, Davies MJ, Prewitt RL, Lauterio TJ. Development of hypertension in a rat model of diet-induced obesity. Hypertension 2000; 35: 1009-1015, doi: 10.1161/01.HYP.35. 4.1009.

20. Nadkarni NA, Chaumontet C, Azzout-Marniche D, Piedcoq J, Fromentin G, Tome D, et al. The carbohydrate sensitive rat as a model of obesity. PLoS One 2013; 8: e68436, doi: 10.1371/journal.pone.0068436.

21. Gerbaix M, Metz L, Ringot E, Courteix D. Visceral fat mass determination in rodent: validation of dual-energy $\mathrm{X}$-ray absorptiometry and anthropometric techniques in fat and lean rats. Lipids Health Dis 2010; 9: 140, doi: 10.1186/1476511X-9-140.

22. Chang S, Graham B, Yakubu F, Lin D, Peters JC, Hill JO. Metabolic differences between obesity-prone and obesityresistant rats. Am J Physiol 1990; 259: R1103-R1110.

23. Commerford SR, Pagliassotti MJ, Melby CL, Wei Y, Hill JO. Inherent capacity for lipogene sis or dietary fat retention is not increased in obesity-prone rats. Am J Physiol Regul Integr Comp Physiol 2001; 280: R1680-R1687.

24. Benevenga NJ, Calvert C, Eckhert CD, Fahey GC, Greger JL, Keen $\mathrm{CL}$, et al. Nutrient requirements of the laboratory rat. In: Anonymous, Nutrient requirements of laboratory animals. Washington: National Academy Press; 1995. p 192.

25. Taylor BA, Phillips SJ. Detection of obesity QTLs on mouse chromosomes 1 and 7 by selective DNA pooling. Genomics 1996; 34: 389-398, doi: 10.1006/geno.1996.0302.

26. Everitt B, Hothorn T. An introduction to applied multivariate analysis with $R$. Heidelberg: Springer; 2011.

27. Assaad H, Yao K, Tekwe CD, Feng S, Bazer FW, Zhou L, et al. Analysis of energy expenditure in diet-induced obese rats. Front Biosci 2014; 19: 967-985.

28. Johnson MM, Peters JP. Technical note: an improved method to quantify nonesterified fatty acids in bovine plasma. J Anim Sci 1993; 71: 753-756.

29. Okere IC, Chandler MP, McElfresh TA, Rennison JH, Sharov V, Sabbah HN, et al. Differential effects of saturated and unsaturated fatty acid diets on cardiomyocyte apoptosis, adipose distribution, and serum leptin. Am J Physiol Heart Circ Physiol 2006; 291: H38-H44, doi: 10.1152/ajpheart.01295.2005.

30. Dourmashkin JT, Chang GQ, Gayles EC, Hill JO, Fried SK, Julien C, et al. Different forms of obesity as a function of diet composition. Int J Obes 2005; 29: 1368-1378, doi: 10.1038/ sj.ijo.0803017.

31. Ebrahim S, Montaner D, Lawlor DA. Clustering of risk factors and social class in childhood and adulthood in British women's heart and health study: cross sectional analysis. BMJ 2004; 328: 861, doi: 10.1136/bmj.38034.702836.55. 
32. Krimer LS, Zaitsev AV, Czanner G, Kroner S, GonzalezBurgos G, Povysheva NV, et al. Cluster analysis-based physiological classification and morphological properties of inhibitory neurons in layers 2-3 of monkey dorsolateral prefrontal cortex. J Neurophysiol 2005; 94: 3009-3022, doi: 10.1152/jn.00156.2005.

33. Pagliassotti MJ, Knobel SM, Shahrokhi KA, Manzo AM, Hill JO. Time course of adaptation to a high-fat diet in obesity-resistant and obesity-prone rats. Am J Physiol 1994; 267: R659-R664.

34. Carroll JF, Zenebe WJ, Strange TB. Cardiovascular function in a rat model of diet-induced obesity. Hypertension 2006; 48: 65-72, doi: 10.1161/01.HYP.0000224147.01024.77.

35. Jackman MR, MacLean PS, Bessesen DH. Energy expenditure in obesity-prone and obesity-resistant rats before and after the introduction of a high-fat diet. Am J Physiol Regul Integr Comp Physiol 2010; 299: R1097-R1105, doi: 10.1152/ajpregu.00549.2009.

36. Boustany CM, Bharadwaj K, Daugherty A, Brown DR, Randall DC, Cassis LA. Activation of the systemic and adipose renin-angiotensin system in rats with diet-induced obesity and hypertension. Am J Physiol Regul Integr Comp Physiol 2004; 287: R943-R949, doi: 10.1152/ajpregu.00265. 2004.

37. Trayhurn P, Beattie JH. Physiological role of adipose tissue: white adipose tissue as an endocrine and secretory organ. Proc Nutr Soc 2001; 60: 329-339, doi: 10.1079/ PNS200194.

38. Levine TB, Levine AB. Metabolic syndrome and cardiovascular disease. Philadelphia: Saunders/Elsevier; 2006.

39. Nascimento AR, Machado M, de Jesus N, Gomes F, Lessa MA, Bonomo IT, et al. Structural and functional microvascular alterations in a rat model of metabolic syndrome induced by a high-fat diet. Obesity 2013; 21: 2046-2054, doi: 10.1002/oby.20358.

40. Reuter S, Bussemaker E, Hausberg M, Pavenstadt $H$, Hillebrand U. Effect of excessive salt intake: role of plasma sodium. Curr Hypertens Rep 2009; 11: 91-97, doi: 10.1007/ s11906-009-0018-5. 